\title{
Lamp Configuration Design for Rapid Thermal Processing Systems
}

\author{
Yaw-Kuen Jan and Ching-An Lin
}

\begin{abstract}
We study lamp configuration design for rapid thermal processing (RTP) systems. We consider a configuration consists of four concentric circular lamp zones, three of them above the wafer and one circumvallating the wafer. We propose a method to determine the geometric parameters, the width, height and radius, of the lamp zones so that the configuration designed has the capacity to achieve uniform temperature on the wafer. The method is based on a necessary and sufficient condition for uniform temperature tracking and analytic expressions of the view factors. A design example is given in which a least square open-loop control law yields good temperature uniformity.
\end{abstract}

Index Terms-Lamp configuration design, rapid thermal processing, tracking, uniform termperature.

\section{INTRODUCTION}

$\mathbf{S}$ INGLE wafer rapid thermal processing (RTP) has become an alternative to the traditional furnace-based batch processing in processes such as titanium silicide formation, implant annealing, nitridation of titanium [1], [2], etc. Maintaining wafer temperature uniformity while following fast temperature trajectories is a key requirement to prevent slip dislocation and to ensure process uniformity, especially during high temperature RTP applications such as rapid thermal oxidation. As the wafer becomes large and the feature size shrinks, whether RTP will become the mainstream processing technology for the next generation of IC fabrication depends very much on how well the wafer temperature can be measured and controlled.

There are many studies in the literature on temperature measurement and temperature control algorithm. Apte et al. [3] has shown that a multiring circularly symmetric lamp configuration with independent (multivariable) control is suitable for RTP system. To optimize the temperature performance, a technique based on convex optimization was developed by Norman [4] with a model based on Lord's work [5]. Schapter et al. [6]-[8] implemented an internal model control (IMC) strategy with gain-scheduling on RTP systems, and Stuber et al. [9] developed a successively linearized quadratic dynamic matrix control (QDMC) strategy. Optical pyrometers are probably the most widely used temperature sensor for RTP systems [10], [11], although acoustic temperature sensors [12],

Manuscript received November 14, 1996; revised July 21, 1997. This work was supported by the National Science Council of the Republic of China under Grant NSC 86-2221-E-009-090.

The authors are with the Department of Control Engineering, National Chiao-Tung University, Hsinchu 300, Taiwan R.O.C. (e-mail: u8312801@cc.nctu.edu.tw; calin@cc.nctu.edu.tw).

Publisher Item Identifier S 0894-6507(98)00341-8.
[13] and wafer expansion technique, e.g., Donnelly et al. [14] are also used.

The most commonly used lamp configurations in RTP system are linear array [2], hexagonal-shaped [15] and concentric circular [16]. However, there are relatively few in-depth studies on lamp configuration design [17].

A good lamp configuration must have the capacity to provide the required radiative heat flux to the wafer surface for maintaining uniform temperature both during steady-state and transient heating. In this paper we consider a lamp configuration consists of four concentric circular lamp zones, three of them above the wafer and one circumvallating the wafer. We propose a method to determine the geometric parameters, the width, height and radius, of the lamp zones so that the configuration designed is best suited for achieving temperature uniformity. The design method is based on a necessary and sufficient condition (on the heat flux distribution on the wafer) for uniformly tracking a desired temperature profile. It turns out that if heat convection is neglected, uniform temperature requires uniform heat flux on the wafer surface and an additional heat flux to compensate for edge loss.

Since heat flux distribution on wafer surface (and on edge) is a positive linear combination of the view factors of the four lamp zones and the view factors due to chamber reflection, it is important that the view factors be properly designed. Clearly, the view factors due to chamber reflection is highly dependent of the lamp configuration. For simplicity, in lamp configuration design we neglect the effect of chamber reflection and consider only the view factors due to the lamps. The effect of chamber reflection is later included in a model for open-loop control law design. To design the lamp view factors, we first derive their analytic expressions (as functions of the geometric parameters) and examine some of the properties. Due to the axisymmetric arrangement of the lamps, the view factors are functions of one variable. It turns out that they are either monotonic decreasing, monotonic increasing, or unimodal concave. The expressions and properties are then used to obtain positive linear combinations which yield uniform heat flux distribution. More precisely, the design is to obtain positive linear combinations that minimize the variation (of the function). With the configuration designed, a least square method is used to compute an open-loop control law (i.e., heat intensities of the four lamp zones) for tracking uniform temperature profiles. In the least square design, the effect due to chamber reflection is included in the dynamic model. Simulation results show that the design and the associated control yield good temperature 


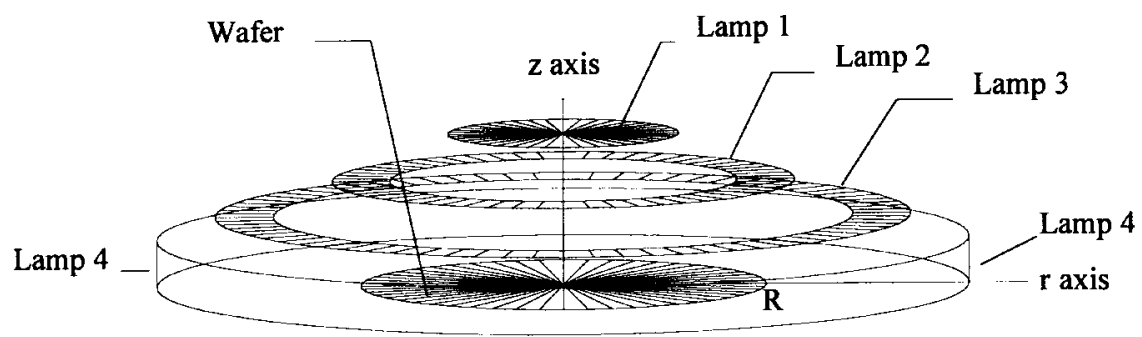

Fig. 1. A simplified schematic of the RTP system.

uniformity and that reflection of the chamber has only a minor effect on the achievable temperature uniformity.

The paper is organized as follows. Section II introduces the RTP system and derives a dynamic model for it. Section III gives a necessary and sufficient condition for uniform temperature tracking. Section IV computes the view factor functions between lamps and wafer and derives some of its properties. Section V gives a lamp configuration design procedure together with a numerical design example. It is shown that open-loop least square control improves temperature uniformity. Section VI is a brief conclusion.

\section{SYSTEM DESCRIPTION}

\section{A. The RTP System Considered}

A simplified schematic of the RTP system is shown in Fig. 1. The system has four concentric circular lamps with three of them above the wafer (lamp 1-3) and one circumvallating the wafer (lamp 4). The bottom edge of lamp 4 is on the same plane as the wafer upper surface. Geometrically, every lamp above the wafer is characterized by its inner radius, width and height; and the lamp circumvallating the wafer is characterized by its radius and width. Unless further specified, units of these geometric parameters are $\mathrm{mm}$. The central axis of the wafer coincides with the central axis of all lamps. The wafer is thin enough so that axial thermal gradient is neglected and the wafer surface is assumed to be gray, diffuse [4]-[6], [17], [18], and opaque [17], [19]. The chamber wall is assumed to be gray, diffuse, and water cooled with temperature much lower than that of the wafer so that heat radiation from it is neglected [6]. Every lamp is assumed to be a black body that emits even heat flux such that view factor approach can be used to describe the radiation phenomenon [5]. Reflection from the chamber wall [20] and the capacitive effect of quartz window [17] are neglected. Since the temperature distribution is axisymmetric, a cylindrical coordinate system is used in which the origin is the center of the wafer upper surface and the $z$-axis coincides with the central axis of the wafer and the lamps.

\section{B. A Dynamic System Model}

This subsection gives a mathematic model describing the wafer temperature dynamics based on the law of energy conservation. Due to the axisymmetry of the RTP system, the surface temperature of each annular wafer zone of infinitesimal width $\delta r$ is assumed to be uniform. Let $T(r, t)(\mathrm{K})$ denote the temperature at time $t$ (second) of the annular wafer zone with inner radius $r$ and outer radius $r+\delta r$. Conservation of energy states that the rate of temperature change in the zone is proportional to the net heat flow rate into it. More precisely,

$$
m C \frac{\partial T}{\partial t}=Q_{k}+Q_{c}+Q_{r}
$$

where $m$ is the mass of the annular zone, $C$ is the specific heat, and $Q_{k}, Q_{c}$, and $Q_{r}$ are heat flow rates into the zone via conduction, convection and radiation, respectively.

Let $G(r, t)(\mathrm{K} / \mathrm{mm})$ be the temperature gradient on $r$. By Fourier's rule of heat conduction, for the annular wafer zone with thickness $H$ inner radius $r$ and outer radius $r+\delta r$,

$$
Q_{k}=-k 2 \pi r H G(r, t)+k 2 \pi[r+\delta r] H G(r+\delta r, t)
$$

where $k$ is the thermal conductivity. Net convective heat into the zone from the surrounding gas of temperature $T_{\infty}(r, t)$ satisfies

$$
Q_{c}=h_{c}(r) \cdot A_{s} \cdot\left[T_{\infty}(r, t)-T(r, t)\right]
$$

where $h_{c}(r)$ is the convective heat transfer coefficient that is a function of $r, A_{s}\left(\mathrm{~mm}^{2}\right)$ is the surface area of the annular zone and to the first order approximation $A_{s}=2 \pi r \cdot \delta r$.

Let

$$
B(r, t)=\sum_{j=1}^{4} F_{j}(r) B_{j}(t)
$$

be the irradiation onto the annular wafer surface due to the lamps, where $B_{j}(t)\left(\mathrm{W} / \mathrm{mm}^{2}\right)$ is the heat flux generated by the $j$ th lamp, $F_{j}(r)$ (no unit) is the radiation view factor from the annular zone to the $j$ th lamp. Since the wafer is opaque with emissivity $\varepsilon$ the net heat flux into the zone is [4], [5]

$$
q(r, t)=\varepsilon\left[B(r, t)-\sigma T(r, t)^{4}\right]
$$

where $\sigma$ is the Stefan-Boltzmann constant $\left(5.67032 \times 10^{-14}\right.$ $\left.\mathrm{W} / \mathrm{mm}^{2} \mathrm{~K}^{4}\right)$ [21]. And the radiation heat into the annular zone is thus

$$
Q_{r}=A_{s} q(r, t) .
$$

With (2.2) through (2.6) and letting $\delta r \rightarrow 0(2.1)$ is rewritten as

$$
\begin{aligned}
\frac{\partial T(r, t)}{\partial t}= & \frac{k}{\rho C r} \frac{\partial[r G(r, t)]}{\partial r}+\frac{h_{c}(r)}{\rho H C}\left[T_{\infty}(r, t)-T(r, t)\right] \\
& +\frac{\varepsilon}{\rho H C}\left[B(r, t)-\sigma T(r, t)^{4}\right]
\end{aligned}
$$


Taking derivative of (2.7) with respect to $r$ gives

$$
\begin{aligned}
\frac{\partial G}{\partial t}= & \frac{k}{\rho C} \frac{\partial^{2} G}{\partial r^{2}}+\frac{k}{\rho C r} \frac{\partial G}{\partial r} \\
& -\left(\frac{k}{\rho C r^{2}}+\frac{\varepsilon \sigma 4 T^{3}}{\rho H C}+\frac{h_{c}}{\rho H C}\right) G \\
& +\frac{\varepsilon}{\rho H C} \frac{\partial B}{\partial r}+\frac{1}{\rho H C}\left[\frac{\partial\left(h_{c} T_{\infty}\right)}{\partial r}-\frac{\partial h_{c}}{\partial r} T\right]
\end{aligned}
$$

where we omit the variables $r$ and $t$.

Since the wafer temperature is uniform if and only if $G(r, t)=0$ for $r \in[0, R),(2.8)$ is used to derive a necessary and sufficient condition on $B(r, t)$ for uniform temperature tracking.

\section{Temperature Uniformity CONDITION}

Suppose the desired temperature trajectory is $T_{d}(t)(\mathrm{K})$ and the wafer temperature is initially uniform at $T_{d}(0)$. If heat exchange through convection on the wafer edge is neglected, then to maintain a uniform temperature $T_{d}(t)$ on the wafer, the irradiation $B_{\text {edge }}(t)\left(\mathrm{W} / \mathrm{mm}^{2}\right)$ that strikes the edge of the wafer must satisfy

$$
B_{\text {edge }}(t)=\sigma T_{d}(t)^{4}
$$

to compensate for radiation heat loss.

From (2.8), if

$$
G(r, t)=0, \quad \text { for all } r \in[0, R) \text { and } t \geq 0
$$
then $\frac{\partial B(r, t)}{\partial r}$ must satisfy

$$
\frac{\partial B(r, t)}{\partial r}=-\frac{1}{\varepsilon}\left\{\frac{\partial\left[h_{c}(r) T_{\infty}(r, t)\right.}{\partial r}-\frac{\partial h_{c}(r)}{\partial r} T(r, t)\right\}
$$

for all $r \in[0, R)$ and $t \geq 0$ in order to retain temperature uniformity. By the axisymmetric arrangement of the RTP system and the assumption that the surface temperature of each annular wafer zone with infinitesimal width $\delta r$ is uniform, no heat conduction occurs at $r=0$ Thus, the desired $B(0, t)$ can be computed from (2.7) by neglecting the first term on the right hand side and substituting $T(r, t)$ by $T_{d}(t)$. That is,

$$
\begin{aligned}
B(0, t)= & \sigma T_{d}(t)^{4}+\frac{1}{\varepsilon}\left\{\rho H C \frac{\partial T_{d}(t)}{\partial t}\right. \\
& \left.-h_{c}(0)\left[T_{\infty}(0, t)-T_{d}(t)\right] .\right\}
\end{aligned}
$$

By integrating (3.3) with $T(r, t)$ substituted by $T_{d}(t)$ and using (3.4), the desired heat flux is given by

$$
\begin{aligned}
B(r, t)= & \sigma T_{d}(t)^{4}+\frac{1}{\varepsilon}\left\{\rho H C \frac{\partial T_{d}(t)}{\partial t}\right. \\
& \left.-h_{c}(r)\left[T_{\infty}(r, t)-T_{d}(t)\right]\right\}
\end{aligned}
$$

for all $r \in[0, R)$. Equation (3.5) gives an explicit formula for the desired heat flux distribution generated by lamps for uniform temperature control.

Thus, (3.1) and (3.5) together give a sufficient and necessary condition for tracking the desired temperature trajectory $T_{d}(t)$ uniformly across the wafer. If heat convection on the wafer surface is neglected, condition (3.5) simplifies to

$$
B(r, t)=\sigma T_{d}(t)^{4}+\frac{1}{\varepsilon} \rho H C \frac{\partial T_{d}(t)}{\partial t}
$$

for all $r \in[0, R)$ and $t \geq 0$.

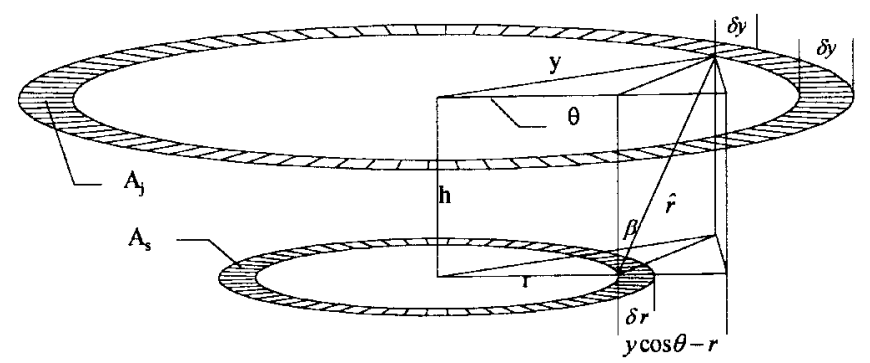

Fig. 2. A simplified schematic for deriving view factor function.

Thus if heat convection is neglected, to maintain uniform temperature on the wafer, we need a uniform heat flux on the wafer surface which depends on both the desired temperature and its rate of change and a heat flux on the wafer edge proportional to the fourth power of the desired temperature. These conditions will be used for the design of lamp configuration. In view of (2.4), expression and some properties of view factors $F_{j}(r)$ are derived next.

\section{THE VIEW FACTOR: Formulas AND PROPERTIES}

Since the heat flux distribution generated by the lamps is given by (2.4), an explicit form of view factor $F_{j}(r)$ as a function of $r$ is useful for the purposes of lamp arrangement and temperature control. In this section we derive explicit formulas for the view factors showing their dependence on the geometric parameters of the lamps, that is, inner radius, width and height. Some properties of the view factors are discussed which is used in Section V for the design of lamp configuration.

\section{A. View Factor on the Wafer Surface}

Each lamp in the RTP system has an associated view factor with the wafer upper surface. Geometrically, there are two kinds of lamps: those above the wafer (lamps 1-3) and that circumvallating the wafer (lamp 4). The associated view factors of lamps 1-3 are derived first.

1) View Factors Associated with Lamps 1-3: A simplified schematic illustrating the geometrical relation between lamp and the annular wafer zone is shown in Fig. 2. The view factor from $A_{s}$ to $A_{j}$ is [5]

$$
F_{j}(r)=\frac{1}{A_{s}} \int_{A_{j}} \int_{A_{s}} \frac{\cos ^{2} \beta}{\pi \hat{r}^{2}} d A_{s} d A_{j}, \quad j=1,2,3
$$

where $A_{j}$ is the surface area $\left(\mathrm{mm}^{2}\right)$ of the $j$ th lamp, $A_{s}$ is the surface area $\left(\mathrm{mm}^{2}\right)$ of the annular wafer zone with inner radius $r$ and width $\delta r, \hat{r}$ is the distance between two infinitesimal elements on $A_{j}$ and $A_{s}$ and $\beta$ is the angle between $z$-axis and the line containing $\hat{r}$. Due to the axisymmetry and the fact that $\delta r$ is infinitesimal, (4.1) simplifies to

$$
F_{j}(r)=\int_{A_{j}} \frac{\cos ^{2} \beta}{\pi \hat{r}^{2}} d A_{j}, \quad j=1,2,3
$$

Let $d_{j}, l_{j}$ and $h_{j}$ be the inner radius, width and height of the $j$ th lamp, respectively. To show the dependence of the view factors on the geometric parameters, we let

$$
f\left(r, d_{j}, h_{j}, l_{j}\right):=F_{j}(r), \quad j=1,2,3 .
$$


By integrating (4.2), ${ }^{1}$

$$
\begin{aligned}
f(r, d, h, l)= & \frac{1}{2} \frac{r^{2}+h^{2}-d^{2}}{\sqrt{\left[(d+r)^{2}+h^{2}\right]\left[(d-r)^{2}+h^{2}\right]}} \\
& +\frac{1}{2} \frac{(d+l)^{2}-h^{2}-r^{2}}{\sqrt{\left[(d+l+r)^{2}+h^{2}\right]\left[(d+l-r)^{2}+h^{2}\right]}} .
\end{aligned}
$$

Some properties of the view factor function $f(r, d, h, l)$ are listed below.

Property 4.1:

$$
\left.\frac{\partial f(r, d, h, l)}{\partial r}\right|_{r=0} \text { and }\left.\quad \frac{\partial f(r, d, h, l)}{\partial r}\right|_{\substack{d=0 \\ r>0}}<0 .
$$

Property 4.2: For each $d>0, h>0, l>0, f(r, d, h, l)$ as a function of $r$ satisfies one of the following.

1) $\frac{\partial f}{\partial r}<0, \forall r \in(0, R)$.

2) $\frac{\partial f}{\partial r}>0, \forall r \in(0, R)$.

3) There exists a unique $r_{0} \in(0, R)$ such that $\frac{\partial f}{\partial r}>0$, $\forall r \in\left(0, r_{0}\right)$ and $\frac{\partial f}{\partial r}<0, \forall r \in\left(r_{0}, R\right)$.

Remarks: a) Property 4.2 says that on $(0, R)$ the view factor function can only be increasing or decreasing or unimodal with a unique local maximum depending on the geometric parameters $d, h$ and $l$. It will become clear that lamp configuration design to satisfy (3.1) and (3.6) amounts to appropriately combining these three types of view factor function (by choosing the geometric parameters and weightings) so as to minimize the variations of the resulting linear combinations. b) For $d=0$, the lamp becomes a disk-type and formula (4.4) reduces to one that can be found in [22].

2) View Factor Associated with Lamp 4: Suppose lamp 4 has radius $d_{4}$ and width $L$. The corresponding view factor on the wafer surface is given by

$$
\begin{aligned}
F_{4}(r)= & \frac{1}{2}+\frac{1}{2} \operatorname{sgn}\left(r^{2}-d_{4}^{2}+L^{2}\right) \\
& \times \sqrt{1-\left[1+\frac{\left(r^{2}-d_{4}^{2}+L^{2}\right)^{2}}{4 d_{4}^{2} L^{2}}\right]^{-1}}
\end{aligned}
$$

It turns out that

$$
\frac{\partial F_{4}(r)}{\partial r}=4 r d_{4}^{2} L^{2} /\left[\left(r^{2}-d_{4}^{2}+L^{2}\right)^{2}+4 d_{4}^{2} L^{2}\right]^{\frac{3}{2}} \geq 0
$$

and hence $F_{4}(r)$ is monotone increasing in $r \in[0, R)$.

\footnotetext{
${ }^{1}$ The integral is evaluated using Mathematica.
}

\section{B. View Factor on the Wafer Edge}

Suppose the edge of the wafer is illuminated only by lamps 3 and 4 (this can be achieved by making the outer radius of lamps 1 and 2 less than $R$ ), we then have

$$
B_{\text {edge }}(t)=F_{e 3} B_{3}(t)+F_{e 4} B_{4}(t) .
$$

We assume that the inner radius of lamp $3, d_{3}$ is larger than $R$.

Since the wafer is thin enough with respect to the wafer radius, we have the following approximation (see (4.7) and (4.8) at the bottom of the page). These two integrals do not depend on the wafer radial coordinate $r$ and can be evaluated by using Mathematica. Note that (4.7) and (4.8) also show the dependence of $F_{e 3}$ and $F_{e 4}$ on the geometric parameters.

\section{LAMP DESIGN FOR UNIFORM TEMPERATURE TRACKING}

\section{A. Constraints on the View Factors}

Equations (3.1) and (3.6) give the desired heat flux $B(r, t)$ and $B_{\text {edge }}(t)$ for wafer temperature to uniformly track $T_{d}(t)$. To satisfy (3.1), we have

$$
F_{e 3} B_{3}(t)+F_{e 4} B_{4}(t)=\sigma T_{d}(t)^{4}
$$

and to satisfy (3.6), we have

$$
\sum_{j=1}^{4} F_{j}(r) B_{j}(t)=\sigma T_{d}(t)^{4}+\frac{1}{\varepsilon} \rho H C \frac{\partial T_{d}(t)}{\partial t} .
$$

The lamp design problem is to determine the geometric parameters of the lamps, which fix $F_{e 3}, F_{e 4}$ and the functions $F_{j}(r)$ so that (5.1) and (5.2) have a solution $B_{j}(t)$. We think of (5.1), for each $t$ as a linear equation on $B_{3}(t)$ and $B_{4}(t)$ with constant coefficients $F_{e 3}$ and $F_{e 4}$. Equation (5.2) is more complicated: for each $t$, it represents a set of infinitely many linear equations, one for each $r$. Given the properties of the view factor functions $F_{j}(r)$, it is unlikely that there exist $F_{j}(r)$ and $B_{j}(t)$ such that (5.2) is satisfied exactly. Our approach to find an approximate solution is to first choose $B_{3}(t)$ and $B_{4}(t)$ as independent variables (and thus $B_{1}(t)$ and $B_{2}(t)$ become dependent variables) and then to design $F_{j}(r)$ so that (5.2) is a linear equation on $B_{3}(t)$ and $B_{4}(t)$ with coefficients which are almost independent of $r$. The variables $B_{j}(t), j=1,2,3,4$, can then be obtained by solving two linear equations.

More precisely, we let

$$
B_{1}(t)=k_{31} B_{3}(t)+k_{41} B_{4}(t)
$$

and

$$
B_{2}(t)=k_{32} B_{3}(t)+k_{42} B_{4}(t)
$$

$$
\begin{aligned}
& F_{e 3}=\frac{1}{\pi} \times \int_{d_{3}}^{d_{3}+l_{3}} \int_{-\cos ^{-1}(R / y)}^{\cos ^{-1}(R / y)} \frac{h_{3}(y \cos \theta-R) y}{\left[y^{2}+R^{2}+h_{3}^{2}-2 y R \cos \theta\right]^{2}} d \theta d y \\
& F_{e 4}=\frac{1}{\pi} \times \int_{0}^{L} \int_{-\cos ^{-1}\left(R / d_{4}\right)}^{\cos ^{-1}\left(R / d_{4}\right)} \frac{\left(d_{4}-R \cos \theta\right)\left(d_{4} \cos \theta-R\right) d_{4}}{\left[y^{2}+R^{2}+d_{4}^{2}-2 d_{4} R \cos \theta\right]^{2}} d \theta d y
\end{aligned}
$$


then (5.2) becomes

$$
F_{u 3}(r) B_{3}(t)+F_{u 4}(r) B_{4}(t)=\sigma T_{d}(t)^{4}+\frac{1}{\varepsilon} \rho H C \frac{\partial T_{d}(t)}{\partial t}
$$

where

$$
F_{u 3}(r)=k_{31} F_{1}(r)+k_{32} F_{2}(r)+F_{3}(r)
$$

and

$$
F_{u 4}(r)=k_{41} F_{1}(r)+k_{42} F_{2}(r)+F_{4}(r)
$$

We think of $F_{u 3}(r)$ and $F_{u 4}(r)$ as equivalent view factors for lamp 3 and lamp 4, respectively. The design is then to make the functions $F_{u 3}$ and $F_{u 4}$ almost constant over $[0, R]$ by choosing the geometric parameters and the coefficients $k_{i j}$, $i=3,4$ and $j=1,2$.

For now we assume that $F_{u 3}$ and $F_{u 4}$ are positive constants (independent of $r$ ), so that (5.1) and (5.3) are two linear equations in two variables. For each $T_{d}(t)$ and $\frac{\partial T_{d}(t)}{\partial t}$, the equations have a solution, which is shown in (5.6) at the bottom of the page, provided $F_{u 3} F_{e 4}-F_{e 3} F_{u 4} \neq 0$. Clearly, the heat flux $B_{3}(t)$ and $B_{4}(t)$ must be positive. A useful sufficient condition for this to hold is the following.

Fact 5.1: Suppose $\frac{\partial T_{d}(t)}{\partial t} \geq 0$ the solution $B_{3}(t)$ and $B_{4}(t)$ in (5.6) are both positive if

$$
F_{e 4}>\left[\frac{\sigma T_{d}(t)^{4}}{\sigma T_{d}(t)^{4}+\frac{\rho H C}{\varepsilon} \frac{\partial T_{d}(t)}{\partial t}}\right] F_{u 4}
$$

and

$$
F_{u 3}>\left[\frac{\sigma T_{d}(t)^{4}+\frac{\rho H C}{\varepsilon} \frac{\partial T_{d}(t)}{\partial t}}{\sigma T_{d}(t)^{4}}\right] F_{e 3} .
$$

Proof: It follows from (5.7) and (5.8) that

$$
F_{u 3} F_{e 4}-F_{e 3} F_{u 4}>0
$$

This together with (5.6) implies that $B_{3}(t)>0$ and $B_{4}(t)>0$ for all $t$

Remarks: a) Another sufficient condition is given by (5.7) and (5.8) with the inequality reversed. This condition is less useful for lamp configuration design. b) From (5.7) it is clear that to hold uniform temperature at steady state, i.e. $\frac{\partial T_{d}(t)}{\partial t}=0$, we must have $F_{e 4}>F_{u 4}$. c) From (5.8) it is clear that $F_{u 3} / F_{e 3}$ is closely related to an upper bound of temperature ramp rate $\frac{\partial T_{d}(t)}{\partial t}$. The larger $F_{u 3} / F_{e 3}$ is, the faster the temperature is allowed to ramp up.

\section{B. Design to Minimize the Variation of View Factors}

We now consider the problem of designing the lamp configuration so that the equivalent view factors $F_{u 3}(r)$ and $F_{u 4}(r)$ defined in the previous subsection are almost independent of $r$, that is, they are almost constant functions. To quantitatively discuss what is meant by "almost independent of $r$ ", we need the notation of the variation of a function on an interval [23].

Definition 5.2 [23]: A partition of an interval $[a, b]$ is a set of points $\left\{x_{0}, x_{1}, \ldots, x_{m}\right\}$ in $[a, b]$ such that $x_{i+1}>x_{i}$, $i=0,1 \ldots, m-1, x_{0}=a$, and $x_{m}=b$.

Definition 5.3 [23]: Let $f$ be a continuous function on $[a, b]$. The variation of function $f$ on $[a, b]$ is defined as

$$
\operatorname{Var}[f ; a, b]=\sup \sum_{i=1}^{m}\left|f\left(x_{i}\right)-f\left(x_{i-1}\right)\right|
$$

where the supremum is taken over all possible partitions of $[a, b]$

Fact 5.4 [23]:

(a) $\operatorname{Var}[f ; a, b] \geq \max _{r \in[a, b]} f(r)-\min _{r \in[a, b]} f(r)$ and the equality holds when $f$ is monotonic.

If $f$ has a continuous derivative $f^{\prime}$ on $[a, b]$, then $\operatorname{Var}[f ; a, b]=\int_{a}^{b}\left|f^{\prime}(r)\right| d r$.

Clearly, $f$ is a constant function if and only if the variation of $f$ is zero. Small variation indicates small deviation from a constant function. Thus our object is to make $\operatorname{Var}\left[F_{u 3} ; 0, R\right]$ and $\operatorname{Var}\left[F_{u 4} ; 0, R\right]$ as small as possible. Note that $F_{u 3}$ and $F_{u 4}$ are, by (5.4) and (5.5), linear combinations of $F_{j}(r)$, $j=1,2,3,4$, which are either decreasing, increasing or unimodal concave. The following results are useful in choosing this class of functions to make the variation of the resulting linear combination small.

Fact 5.5: Let the functions $f_{1}(r)$ and $f_{2}(r)$ be, respectively, monotone decreasing and monotone increasing on $[a, b]$ with continuous derivatives $f_{1}^{\prime}(r)$ and $f_{2}^{\prime}(r)$. Let

$$
C=\left\{r \in[a, b]|| f_{1}^{\prime}(r)|>| f_{2}^{\prime}(r) \mid\right\} .
$$

If

$$
\operatorname{Var}\left[f_{1} ; a, b\right]=\operatorname{Var}\left[f_{2} ; a, b\right]
$$

then

$$
\operatorname{Var}\left[f_{1}+f_{2} ; a, b\right]=2 \int_{C}\left[\left|f_{1}^{\prime}(r)\right|-\left|f_{2}^{\prime}(r)\right|\right] d r
$$

Remark: Thus the sum of a monotone decreasing function and a monotone increasing one gives smaller variation if they have equal variations. Moreover, the variation of the sum is zero if the set $C$ is empty.

In the lamp configuration proposed, we have $d_{1}=0$ and thus $\frac{\partial F_{1}(r)}{\partial r} \leq 0$, i.e. $F_{1}(r)$ is monotone decreasing

$$
\left\{\begin{array}{l}
B_{3}(t)=\frac{1}{F_{e 4} F_{u 3}-F_{u 4} F_{e 3}}\left[\left(F_{e 4}-F_{u 4}\right) \sigma T_{d}(t)^{4}+F_{e 4} \frac{\rho H C}{\varepsilon} \frac{\partial T_{d}(t)}{\partial t}\right] \\
B_{4}(t)=\frac{1}{F_{e 4} F_{u 3}-F_{u 4} F_{e 3}}\left[\left(F_{u 3}-F_{e 3}\right) \sigma T_{d}(t)^{4}-F_{e 3} \frac{\rho H C}{\varepsilon} \frac{\partial T_{d}(t)}{\partial t}\right]
\end{array}\right.
$$



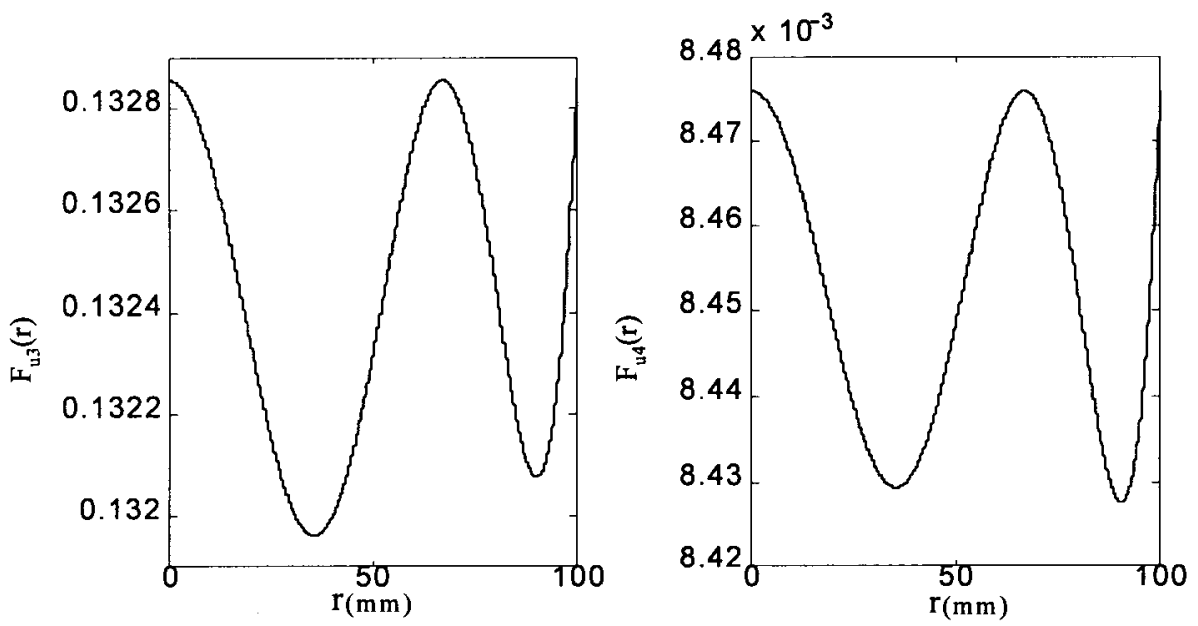

Fig. 3. Calculated view factors, $F_{u 3}(r)$ and $F_{u 4}(r)$, for the case of $k_{31}=k_{32}=1$.

in $[0, R]$. Hence to produce a $F_{u 3}(r)$, in (5.4), of small variation we first find $k_{31} F_{1}(r)+F_{3}(r)$ of smaller variation by choosing monotone increasing $F_{3}(r)$ and $k_{31}$ such that $\operatorname{Var}\left[k_{31} F_{1} ; 0, R\right]=\operatorname{Var}\left[F_{3} ; 0, R\right]$. If $\operatorname{Var}\left[k_{31} F_{1}+F_{3} ; 0, R\right] \neq$ 0 , we need a unimodal and concave $F_{2}(r)$ to further reduce the variation. This is done by suitably adjusting peak location $r_{0}$ of $F_{2}(r)$ to match the peak location of $k_{31} F_{1}(r)+F_{3}(r)$ and choosing $k_{32}$ such that

$$
\operatorname{Var}\left[k_{32} F_{2} ; 0, r_{0}\right]=\operatorname{Var}\left[k_{31} F_{1}+F_{3} ; 0, r_{0}\right] .
$$

In designing $F_{u 3}(r)$ of small variation by finding $h_{i}, l_{i}$ and $d_{j}>0, i=1,2,3, j=2,3$ and $k_{31}, k_{32}>0$, the equations describing those requirements are summarized as follows:

$$
\begin{aligned}
& F_{2}(0)=F_{2}(R) \\
& k_{31}\left[F_{1}(0)-F_{1}(R)\right]=F_{3}(R)-F_{3}(0) \\
& \underset{r \in[0, R]}{\operatorname{Arg} \operatorname{Min}\left[k_{31} F_{1}(r)+F_{3}(r)\right]}=\underset{r \in[0, R]}{\operatorname{Arg} \operatorname{Max} F_{2}(r) \triangleq r_{0}} \\
& {\left[k_{31} F_{1}(0)+F_{3}(0)\right]-\left[k_{31} F_{1}\left(r_{0}\right)+F_{3}\left(r_{0}\right)\right] } \\
&=k_{32}\left[F_{2}\left(r_{0}\right)-F_{2}(0)\right]
\end{aligned}
$$

subject to

$$
\frac{\partial F_{3}(r)}{\partial r}>0, \quad \text { for } r \in(0, R) .
$$

To consider the design of view factor function $F_{4}(r)$ we first note that by (4.6), $F_{4}(r)$ is monotone increasing. Once parameters of lamps 1 and 2 have been determined from (5.9) and (5.10), $F_{u 4}(r)$ of small variation can be obtained following the same design approach above by finding $d_{4}, L$ and $k_{41}$ such that

$$
\left\{\begin{array}{l}
k_{41}\left[F_{1}(0)-F_{1}(R)\right]=F_{4}(R)-F_{4}(0) \\
\operatorname{Arg} \operatorname{Min}_{r \in[0, R]}\left[k_{41} F_{1}(r)+F_{4}(r)\right]=r_{0} .
\end{array}\right.
$$

The parameter $k_{42}$ is then computed by

$$
\begin{aligned}
k_{42}= & \left\{\left[k_{41} F_{1}(0)+F_{4}(0)\right]-\left[k_{41} F_{1}\left(r_{0}\right)+F_{4}\left(r_{0}\right)\right]\right\} / \\
& {\left[F_{2}\left(r_{0}\right)-F_{2}(0)\right] . }
\end{aligned}
$$

Note that there are 14 design parameters for lamps 1 through 4 and we have seven equality constraints listed in (5.9), (5.11), and (5.12). Hence, a procedure to obtain a unique design allows some parameters such as $k_{31}, k_{32}, h_{1}, l_{1}, l_{2}, l_{3}$, and $L$ to be fixed and leaves seven parameters $d_{2}, h_{2}, d_{3} h_{3}, d_{4}, k_{41}$, and $k_{42}$ to be found. Although any parameter can be chosen fixed, physical constraints have to be taken into consideration in making the choices. For example, if it is desirable to have lamps of compatible sizes, then the parameters $l_{1}, l_{2}, l_{3}$, and $L$ should be chosen fixed.

\section{Design Procedure and Examples}

Let $H$ and $D$ be the maximal allowable distance from lamp to wafer and inner radius of a lamp in the RTP system, respectively. We summarize the following design procedure for the case where $h_{1}, l_{1}, l_{2}, l_{3}$, and $L$ are fixed and $k_{31}=$ $k_{32}=1$.

(S1) Decrease $h_{2}$ from $H$. For each $h_{2}$ find $d_{2}$, the inner radius of lamp 2, so that the corresponding view factor function $F_{2}(r)$ satisfies $F_{2}(0)=F_{2}(R)$ and record the location at which $F_{2}(r)$ reaches maximum.

(S2) Decrease $h_{3}$ from $H$. For each $h_{3}$ find $d_{3}$, the inner radius of lamp 3 , so that the monotonicity condition (5.10) is satisfied and $\operatorname{Var}\left[F_{1} ; 0, R\right]=\operatorname{Var}\left[F_{3} ; 0, R\right]$. Record the location at which $F_{1}(r)+F_{3}(r)$ reaches minimum.

(S3) From the sets found in (S1) and (S2), choose the combination of $F_{3}(r)+F_{1}(r)$ and $F_{2}(r)$ such that $\operatorname{Var}\left[F_{1}+F_{3} ; 0, R\right]=\operatorname{Var}\left[F_{2} ; 0, R\right]$ and the minimum of $F_{3}(r)+F_{1}(r)$ occurs at the same location as the maximum of $F_{2}(r)$ does.

(S4) Find $F_{4}(r)$ and $k_{41}$ such that $\operatorname{Var}\left[k_{41} F_{1} ; 0, R\right]=\operatorname{Var}\left[F_{4} ; 0, R\right]$ and the peak of $k_{41} F_{1}(r)+F_{4}(r)$ occurs at the same $r_{0}$ as where the peak of $F_{2}(r)$ occurs.

(S5) Compute $k_{42}$ from (5.12).

(S6) Find the upper bound of $\frac{\partial T_{d}(t)}{\partial t}$ by using (5.8) and make sure that it is large enough.

Remark: In the design steps (S1), (S3), and (S4), we have to make sure that the radiation of each lamp on the wafer surface is not blocked by the others. 


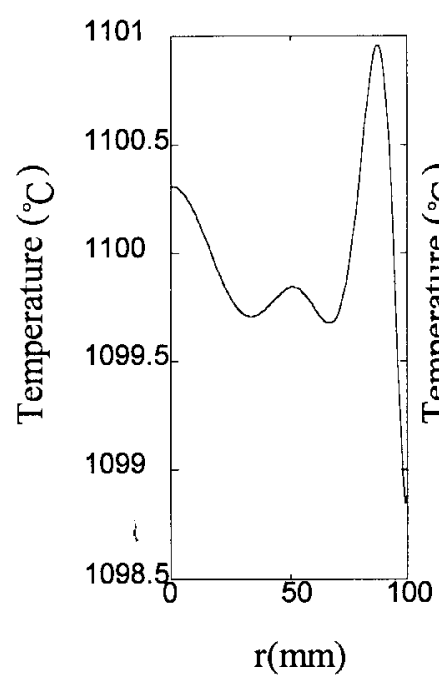

(a)

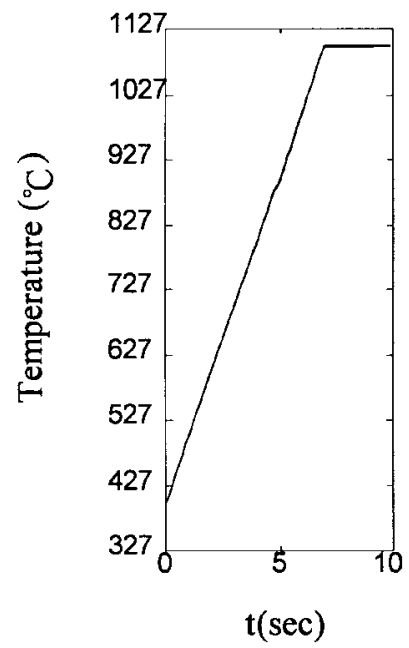

(d)

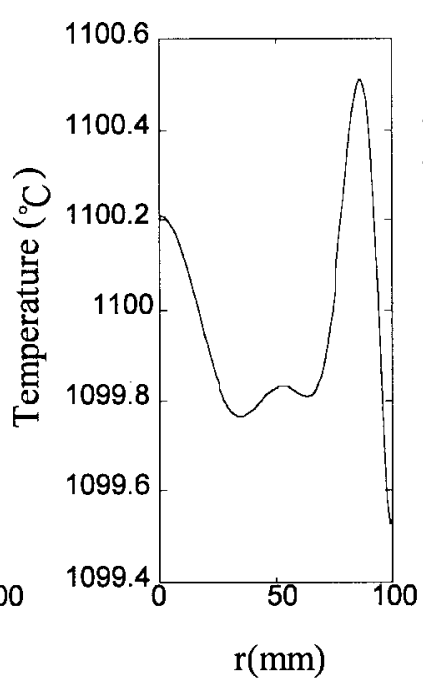

(b)

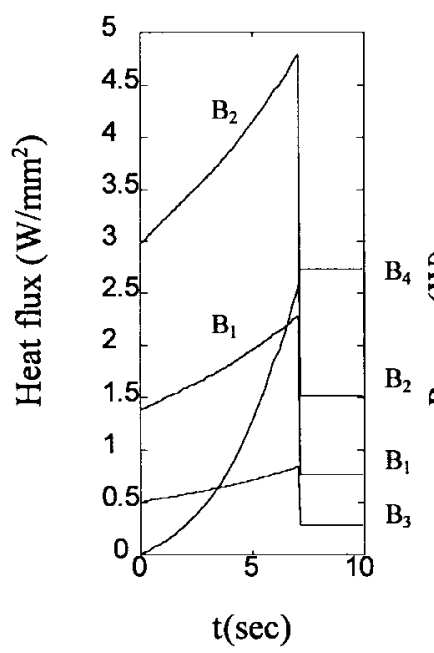

(e)

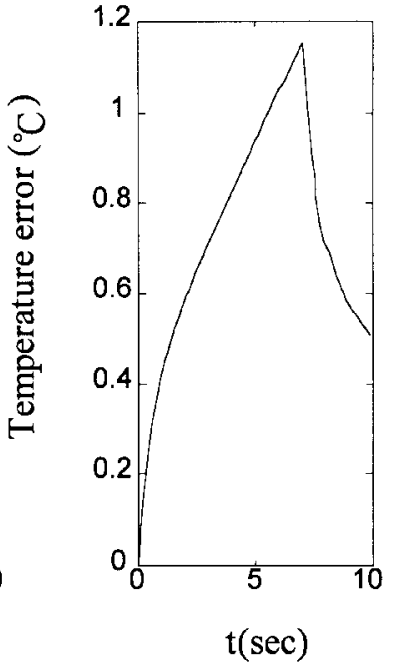

(c)

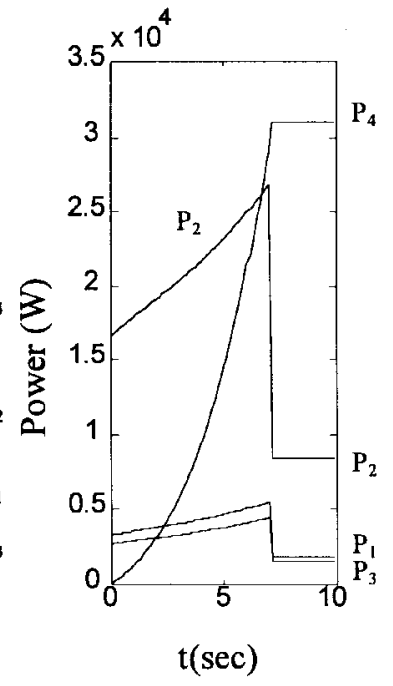

(f)

Fig. 4. Simulation results of type 7 with minimal square error open-loop control law [where $\rho_{w}=0.5$ and $\left.T_{d}(r, t)=T_{A}(t)\right]$. (a) $T(r, 7)$, (b) $T(r, 10)$, (c) $\operatorname{Max}_{r \in[0,100]}\left|T(r, t)-T_{d}(r, t)\right|$, (d) $T(r, t) \quad r=0,1, \ldots, 100$, (e) $B_{j}(t) j=1,2,3,4$, and (f) power needed for each lamp.

The following example illustrates the design procedure. The widths of the ring-type lamps and lamp 4 are fixed at 10 $\mathrm{mm}$ and the radius of the disk-type lamp 1 is $25 \mathrm{~mm}$. The maximal allowable distance from lamp to wafer is $100 \mathrm{~mm}$ and the radius of the chamber is $200 \mathrm{~mm}$. The Silicon wafer is a disk with radius $100 \mathrm{~mm}$ and thickness $1 \mathrm{~mm}$. Under these assumptions, the solution found by numerical approximation is $d_{3}=135.8 \mathrm{~mm}, h_{3}=49.6 \mathrm{~mm}, d_{2}=77.16 \mathrm{~mm}, h_{2}=73.2$ $\mathrm{mm}, d_{4}=194.5 \mathrm{~mm}, k_{41}=0.0515$ and $k_{42}=0.0468$. The equivalent view factors $F_{u 3}(r)$ and $F_{u 4}(r)$ are plotted in Fig. 3. Note that the ratio $\frac{\max _{r \in[0,100]} F_{u i}(r)-\min _{r \in[0,100]} F_{u i}(r)}{F_{u i}(0)}$ is less than 0.006 for $i=3,4$. However the maximal allowable heating ramp $\frac{\partial T_{d}(t)}{\partial t}$ of this design [computed by (5.8)] is only $11^{\circ} \mathrm{C} / \mathrm{s}$ at $400{ }^{\circ} \mathrm{C}$, thus it is not a good design.

It follows from (5.8) that to have large $\frac{\partial T_{d}(t)}{\partial t}$ we must have $F_{u 3}$ large, and from (5.4) one way to achieve this is to have $F_{3}(r)$ large. Now since $F_{3}(r)$ is required to be monotone increasing on $[0, R]$ large $F_{3}(r)$ can be obtained if we choose $d_{3}$ close to $R$. Hence instead of fixing $k_{31}$ and $k_{32}$ to 1 , we fix $d_{3}$ and $h_{3}$. Table I lists nine different designs for different choices of $d_{3}$ and $h_{3}$, the other fixed parameters are $d_{1}=0$, $h_{1}=100 \mathrm{~mm}, l_{1}=25 \mathrm{~mm}$, and $l_{2}=l_{3}=L=10 \mathrm{~mm}$. Simulations are performed for each of the nine designs and the upper bound of $\frac{\partial T_{d}(t)}{\partial t}$ for each configurations is calculated using (5.8). For the ramp-up case, the starting temperature is $400{ }^{\circ} \mathrm{C}$ and the final steady-state temperature is $1100{ }^{\circ} \mathrm{C}$. Two temperature profiles are considered where profile $A$ is defined as

$$
\begin{aligned}
T_{A}(0) & =400^{\circ} \mathrm{C},\left.\frac{\partial T_{A}(t)}{\partial t}\right|_{t \in[0,7)} \\
& =100^{\circ} \mathrm{C} / \mathrm{s},\left.T_{A}(t)\right|_{t \in[7,20]}=1100^{\circ} \mathrm{C}
\end{aligned}
$$

and profile $B$ is defined as

$$
\begin{aligned}
T_{B}(0) & =400^{\circ} \mathrm{C},\left.\frac{\partial T_{B}(t)}{\partial t}\right|_{t \in[0,14)} \\
& =50^{\circ} \mathrm{C} / \mathrm{s},\left.T_{B}(t)\right|_{t \in[14,30]}=1100^{\circ} \mathrm{C} .
\end{aligned}
$$


TABLE I

SOLUTIONS FOUND FOR $d_{1}=0, h_{1}=100 \mathrm{~mm}, l_{1}=25 \mathrm{~mm}$, and $l_{2}=l_{3}=L=10 \mathrm{~mm}$

\begin{tabular}{c|c|c|c|c|c|c|c|c|c|c}
\hline $\begin{array}{c}\text { Lamp } \\
\text { Type }\end{array}$ & $d_{3}(\mathrm{~mm})$ & $h_{3}(\mathrm{~mm})$ & $d_{2}(\mathrm{~mm})$ & $h_{2}(\mathrm{~mm})$ & $r_{0}(\mathrm{~mm})$ & $d_{4}(\mathrm{~mm})$ & $k_{31}$ & $k_{32}$ & $k_{41}$ & $k_{42}$ \\
\hline 1 & 97 & 26 & 67.2566 & 48.89 & 65.3917 & 246.25 & 4.1743 & 1.6442 & 0.0165 & 0.0043 \\
\hline 2 & 97 & 24 & 76.5996 & 71.96 & 66.8311 & 195.95 & 4.5520 & 5.5661 & 0.0496 & 0.0425 \\
\hline 3 & 97 & 23 & 83.9923 & 87.5 & 67.5715 & 180.8 & 4.7607 & 11.5470 & 0.0753 & 0.1316 \\
\hline 4 & 98 & 24 & 84.4422 & 88.4 & 67.609 & 180.13 & 4.5239 & 11.2532 & 0.0768 & 0.1396 \\
\hline 5 & 98 & 26 & 71.8002 & 60.8 & 66.1849 & 213.84 & 4.1567 & 2.9491 & 0.0301 & 0.0146 \\
\hline 6 & 100 & 28 & 72.3756 & 62.2 & 66.2713 & 211.1 & 3.7693 & 2.7924 & 0.0342 & 0.0180 \\
\hline 7 & 100 & 26 & 84.1770 & 87.87 & 67.587 & 180.53 & 4.0696 & 9.6751 & 0.0759 & 0.1348 \\
\hline 8 & 110 & 35 & 79.6668 & 78.6 & 67.1692 & 188.5 & 2.4858 & 3.6058 & 0.0605 & 0.0709 \\
\hline 9 & 110 & 37 & 72.4916 & 62.48 & 66.2885 & 210.54 & 2.3734 & 1.6131 & 0.0347 & 0.0185 \\
\hline
\end{tabular}

TABLE II

Associated Simulation Data for the Lamp Designed

\begin{tabular}{c|c|c|c|c|c|c|c|c|c}
\hline $\begin{array}{c}\text { Lamp } \\
\text { Type }\end{array}$ & $\begin{array}{c}\text { MRP } \\
\left({ }^{\circ} \mathrm{C} / \mathrm{sec}\right)\end{array}$ & $\begin{array}{c}\text { Err1 } \\
\left({ }^{\circ} \mathrm{C}\right)\end{array}$ & $\begin{array}{c}\text { Err2 } \\
\left({ }^{\circ} \mathrm{C}\right)\end{array}$ & $\begin{array}{c}\text { Err3 } \\
\left({ }^{\circ} \mathrm{C}\right)\end{array}$ & $\begin{array}{c}\text { Err4 } \\
\left({ }^{\circ} \mathrm{C}\right)\end{array}$ & $\begin{array}{c}\text { Err5 } \\
\left({ }^{\circ} \mathrm{C}\right)\end{array}$ & $\begin{array}{c}\text { Err6 } \\
\left({ }^{\circ} \mathrm{C}\right)\end{array}$ & $\begin{array}{c}\mathrm{P}_{\mathrm{A}} \\
(\mathrm{kW})\end{array}$ & $\begin{array}{c}\mathrm{P}_{\mathrm{B}} \\
(\mathrm{kW})\end{array}$ \\
\hline 1 & 112 & 13.0591 & 6.4908 & 10.7065 & 6.2248 & 4.7527 & 0.4201 & 109.19 & 98.453 \\
\hline 2 & 163 & 12.0450 & 5.7309 & 9.8351 & 5.4609 & 4.0915 & 0.3678 & 79.312 & 66.959 \\
\hline 3 & 226 & 8.8860 & 3.9106 & 7.1367 & 3.6950 & 2.7499 & 0.2470 & 78.230 & 63.444 \\
\hline 4 & 181 & 7.3934 & 3.2099 & 5.9207 & 3.0282 & 2.2518 & 0.2022 & 78.415 & 63.413 \\
\hline 5 & 104 & 11.1584 & 5.4415 & 9.1434 & 5.2011 & 3.9235 & 0.3508 & 87.274 & 75.877 \\
\hline 6 & 70 & NA & NA & 6.0383 & 3.3834 & 2.5591 & 0.2278 & NA & 74.031 \\
\hline 7 & 118.7 & 5.4234 & 2.3202 & 4.3282 & 2.1853 & 1.6254 & 0.1458 & 78.359 & 63.352 \\
\hline 8 & 33.7 & NA & NA & NA & NA & NA & NA & NA & NA \\
\hline 9 & 24.4 & NA & NA & NA & NA & NA & NA & NA & NA \\
\hline
\end{tabular}

MRP denotes maximal allowable heating ramp $\left({ }^{\circ} \mathrm{C} / \mathrm{sec}\right)$ at $400^{\circ} \mathrm{C}$,

Err1 $=\underset{r \in[0,100], t \in[0,7)}{\operatorname{Max}}\left|T(r, t)-T_{d}(r, t)\right|$ and Err2= $\underset{r \in[0,100]}{\operatorname{Max}}\left|T(r, 20)-T_{d}(r, 20)\right|$ with $T_{d}(r, t)=T_{A}(t)$,

Err3 $=\underset{r \in[0,100], t \in[0,14)}{\operatorname{Max}}\left|T(r, t)-T_{d}(r, t)\right|$ and Err4 $=\underset{r \in[0,100]}{\operatorname{Max}}\left|T(r, 30)-T_{d}(r, 30)\right|$ with $T_{d}(r, t)=T_{B}(t)$,

Err5 $=\underset{r \in[0,100], t \in[0,7]}{\operatorname{Max}}\left|T(r, t)-T_{d}(r, t)\right|$ with $T(r, 0)=T_{d}(r, t)=1100^{\circ} \mathrm{C}$,

Err6 $=\underset{r \notin 0,100], t \in[0,7]}{\operatorname{Max}}\left|T(r, t)-T_{d}(r, t)\right|$ with $T(r, 0)=T_{d}(r, t)=400^{\circ} \mathrm{C}, \mathrm{P}_{\mathrm{A}}$ denotes maximal power $(\mathrm{kW})$

needed when $T_{d}(r, t)=T_{A}(t)$, and $\mathrm{P}_{\mathrm{B}}$ denotes maximal power $(\mathrm{kW})$ needed when $T_{d}(r, t)=T_{B}(t)$.

At time $t$ the tracking error for temperature profile $T_{d}(t)$ is defined as $\operatorname{Max}_{r \in[0,100]}\left|T(r, t)-T_{d}(r, t)\right|$. The maximal allowable ramp rate, tracking errors, and maximal power needed for different temperature profiles are listed in Table II. The results show that the configuration type 7 with minimal dynamic tracking error and a heating ramp of $118.7^{\circ} \mathrm{C} / \mathrm{s}$ is the best among the designs. The temperature performance is further improved by a least square-based open-loop control discussed in the next subsection.

In simulation, a silicon wafer with diameter $200 \mathrm{~mm}$ is equally divided into 200 concentric zones. The temperature on each of these 200 concentric zones is assumed to be uniform. Variable-Step-Kutta-Merson integration method [24] is used to simulate wafer temperature dynamics. Since the parameters $k$ and $\rho C$ are temperature dependent, polynomial curve fitting based on the discrete data given in [25] is used to obtain

$$
\begin{aligned}
k= & 0.575872-\frac{7.8427 \times 10^{8}}{T^{3}}+\frac{4.78671 \times 10^{6}}{T^{2}} \\
& +\frac{3.10482 \times 10^{4}}{T}\left(\text { unit } \frac{\mathrm{W}}{\mathrm{m} \cdot{ }^{\circ} \mathrm{K}}\right)
\end{aligned}
$$

and

$$
\begin{aligned}
\frac{k}{\rho C}= & 0.65765 \times 10^{-15}-\frac{1.66045 \times 10^{3}}{T^{3}}+\frac{13.6744}{T^{2}} \\
& -\frac{3.755 \times 10^{-13}}{T}\left(\text { unit } \frac{\mathrm{m}^{2}}{\mathrm{sec}}\right) .
\end{aligned}
$$

\section{Least Square Open-Loop Control}

By independently controlling these four lamps, several lamp configurations found in previous subsection have the 
TABLE III

Maximal Tracking Errors $\left({ }^{\circ} \mathrm{C}\right)$ for Profile A by Using the Open-Loop Control LaW

\begin{tabular}{|c|c|c|c|c|c|c|c|c|}
\hline & $\rho_{w}=0$ & $\rho_{w}=0.1$ & $\rho_{w}=0.3$ & $\rho_{w}=0.5$ & $\rho_{w}=0.57$ & $\rho_{w}=0.65$ & $\rho_{w}=0.8$ & $\rho_{w}=1$ \\
\hline type 1 & 4.1347 & 3.9489 & 3.6214 & 3.3428 & 3.2555 & 3.1616 & 3.0015 & 3.1246 \\
\hline type 2 & 1.3983 & 1.7113 & 2.2533 & 2.7014 & 2.8393 & 2.9861 & 3.2332 & 3.5123 \\
\hline type 3 & 0.9327 & 1.0914 & 1.3632 & 1.5861 & 1.6546 & 1.7277 & 1.8511 & 1.9920 \\
\hline type 4 & 0.7643 & 0.9137 & 1.1707 & 1.3825 & 1.4479 & 1.5178 & 1.6363 & 1.7725 \\
\hline type 5 & 1.7717 & 1.8532 & 2.3290 & 2.8281 & 2.9850 & 3.1539 & 3.4427 & 3.7764 \\
\hline type 7 & 0.5601 & 0.7030 & 0.9497 & 1.1540 & 1.2171 & $*$ & * & * \\
\hline \multicolumn{9}{|c|}{$\begin{array}{l}\text { Each entry indicates } \underset{r \in[0,100], t \in[0,20]}{\operatorname{Max}}\left|T(r, t)-T_{d}(r, t)\right| \text { with } T_{d}(r, t)=T_{A}(t) \text {. } \\
\text { * The least square solution is not feasible. }\end{array}$} \\
\hline
\end{tabular}

capability of maintaining a reasonably uniform temperature over a range of operating conditions. In this subsection, we consider an open-loop control based on the minimization of square error. The effect of reflection due to the circular chamber is included in the model. In this method, the heat flux vector $\left[\begin{array}{llll}B_{1}(t) & B_{2}(t) & B_{3}(t) & B_{4}(t)\end{array}\right]^{T}$ is obtained by finding the least square solution to a linear equation. By finite difference approximation to the heat equation, a model in vector matrix form [4] is given by

$$
\left[\begin{array}{c}
\dot{T}_{1} \\
\vdots \\
\dot{T}_{N}
\end{array}\right]=D\left[\begin{array}{c}
T_{1} \\
\vdots \\
T_{N}
\end{array}\right]+E\left[\begin{array}{c}
T_{1}^{4} \\
\vdots \\
T_{N}^{4}
\end{array}\right]+F\left[\begin{array}{c}
B_{1} \\
B_{2} \\
B_{3} \\
B_{4}
\end{array}\right]
$$

where the wafer is divided into $N$ concentric zones and the radiosity over each zone is assumed to be uniform, $T_{i}$, $i=1,2, \ldots, N-1$, is the temperature of the $i$ th wafer zone, $T_{N}$ is the temperature of wafer edge, matrix $D$ describes the effect of conduction, and matrices $E$ and $F$ describe the effect of radiation including the chamber wall reflection.

Given the desired uniform temperature $T_{d}(t)$, (5.13) becomes

$$
F\left[\begin{array}{c}
B_{1} \\
B_{2} \\
B_{3} \\
B_{4}
\end{array}\right]=\left[\begin{array}{c}
\dot{T}_{d} \\
\vdots \\
\dot{T}_{d}
\end{array}\right]-D\left[\begin{array}{c}
T_{d} \\
\vdots \\
T_{d}
\end{array}\right]-E\left[\begin{array}{c}
T_{d}^{4} \\
\vdots \\
T_{d}^{4}
\end{array}\right]
$$

where the second term on the right hand side is zero. For $N=201$, this is an overdetermined linear equation with four unknowns. The least square solution is given by

$$
\left[\begin{array}{c}
B_{1} \\
B_{2} \\
B_{3} \\
B_{4}
\end{array}\right]=\left(F^{T} F\right)^{-1} F^{T}\left\{\left[\begin{array}{c}
\dot{T}_{d} \\
\vdots \\
\dot{T}_{d}
\end{array}\right]-E\left[\begin{array}{c}
T_{d}^{4} \\
\vdots \\
T_{d}^{4}
\end{array}\right]\right\}
$$

where $F^{T}$ denotes the transpose of $F$.

This control law is applied to the lamp configurations listed in Table I except types 6, 8, and 9 (since the least square solutions of these three types are not feasible). For the temperature profile $A$ (which has a temperature ramp of 100 ${ }^{\circ} \mathrm{C} / \mathrm{s}$ ), maximal tracking errors associated with different wall reflectivity, $\rho_{w}$, are listed in Table III. Note that types 1-5 have nonnegative least square solutions for $\rho_{w} \in[0,1]$ and type 7 does for $\rho_{w} \in[0,0.57]$. The maximal tracking error is less than $1.22{ }^{\circ} \mathrm{C}$ with type 7 for $\rho_{w} \in[0,0.57]$ and it is less than 1.78 ${ }^{\circ} \mathrm{C}$ with type 4 for $\rho_{w} \in[0,1]$. For the types shown, except for type 1, temperature uniformity deteriorates as the chamber reflectivity increases. The increase in maximal tracking error, however, is not drastic. From Table III, it seems that types 3, 4 and 7 are better designs. The temperature responses (with unit degrees Celsius), heat flux, and lamp power of type 7 are plotted on Fig. 4 for the case of $\rho_{w}=0.5$. In this case, the maximal tracking error is less than $1.16^{\circ} \mathrm{C}$.

\section{CONCLUSION}

We have proposed a lamp configuration design method for achieving temperature uniformity of a RTP system. The basic idea is to design the view factors associated with the lamps so as to minimize the variation of the flux distribution on the wafer surface. In developing the design procedure, we have made a number of simplifying assumptions: every lamp is a black body; the wafer is gray, diffuse and opaque; heat convection, the capacitive effect of quartz window and the effect of chamber reflection are neglected. These assumptions together with the analytic expressions of the lamp view factors made the design procedure analytic in nature. The effectiveness of the design method is demonstrated through a design example in which several configurations are obtained. We evaluate the designs with least square control using a model that includes chamber reflection effect. It turns out that with a $200-\mathrm{mm}$ wafer subjected to $100{ }^{\circ} \mathrm{C} / \mathrm{s}$ temperature ramp, some of the designs have maximal temperature deviation in the vicinity of $1{ }^{\circ} \mathrm{C}$ under various chamber reflectivity conditions. The lamp configurations designed are reasonably good, at least under this ideal condition. In a practical setting, it would be necessary to do experiment with both open-loop and feedback control to evaluate the merit of the lamp design.

\section{REFERENCES}

[1] L. Peters, "The hottest topic in RTP," Semicond. Int., pp. 56-62, Aug. 1991.

[2] P. Singer, "Rapid thermal processing: A progress report," Semicond. Int., pp. 64-69, May 1993.

[3] P. P. Apte and K. C. Saraswat, "Rapid thermal processing uniformity using multivariable control of a circularly symmetric 3 zone lamp," IEEE Trans. Semiconduct. Manufact., vol. 5, no. 3, pp. 180-188, Aug. 1992. 
[4] S. A. Norman, "Wafer temperature control in rapid thermal processing," Ph.D. dissertation, Stanford Univ., Stanford, CA, 1992.

[5] H. A. Lord, "Thermal and stress analysis of semiconductor wafers in a rapid thermal processing oven," IEEE Trans. Semiconduct. Manufact., vol. 1, pp. 105-114, Aug. 1988

[6] C. D. Schaper, M. M. Moslehi, K. C. Saraswat, and T. Kailath, "Modeling, identification, and control of rapid thermal processing systems," J. Electrochem. Soc., vol. 141, no. 11, pp. 3200-3209, Nov. 1994.

[7] C. D. Schaper, "Real-time control of rapid thermal processing semiconductor manufacturing equipment," in Proc. American Control Conf., June 1993, pp. 2985-2989.

[8] C. Schaper, M. Moslehi, K. Saraswat, and T. Kailath, "Control of MMST RTP: Repeatability, uniformity, and integration for flexible manufacturing," IEEE Trans. Semiconduct. Manufact., vol. 7, pp. 202-219, May 1994.

[9] J. D. Stuber, T. F. Edgar, J. K. Elliott, and T. Breedijk, "Model-based control of rapid thermal processes," in Proc. IEEE 33rd Conf. Decision and Control, Dec. 1994, pp. 79-85.

[10] F. Roozeboom and N. Parekh, "Rapid thermal processing systems: A review with emphasis on temperature control," J. Vac. Sci. Technol., vol B8, no. 6, pp. 1249-1259, Nov./Dec. 1990.

[11] F. Y. Sorrell, S. Yu, and W. J. Kiether, "Applied RTP optical modeling: An argument for model-based control," IEEE Trans. Semiconduct. Manufact., vol. 7, pp. 454-459, Nov. 1994.

[12] F. L. Degertekin, J. Pei, B. T. Khuri-Yakub, and K. C. Saraswat, "In situ acoustic temperature tomography of semiconductor wafers," Appl. Phys. Lett., vol. 64, no. 11, pp. 1338-1340, Mar. 1994.

[13] Y. J. Lee, B. T. Khuri-Yakub, and K. Saraswat, "Temperature measurement in rapid thermal processing using the acoustic temperature sensor," IEEE Trans. Semiconduct. Manufact., vol. 9, pp. 115-121, Feb. 1996.

[14] V. M. Donnelly and J. A. McCaulley, "Infrared-laser interferometric thermometry: A nonintrusive technique for measuring semiconductor wafer temperatures," J. Vac. Sci. Technol., vol. A8, no. 1, pp. 84-92, Jan./Feb. 1990

[15] B. J. Cho, P. Vandenabeele, and K. Maex, "Development of a hexagonalshaped rapid thermal processor using a vertical tube," IEEE Trans. Semiconduct Manufact., vol. 7, pp. 345-353, Aug. 1994.

[16] J. P. Zöllner, K. Ullrich, J. Peroldt, and G. Eichhorn, "New lamp arrangement for rapid thermal processing," Appl. Surf. Sci., vol. 69, pp. 193-197, 1993

[17] Y. M. Cho, A. Paulraj, T. Kailath, and G. Xu, "A contribution to optimal lamp design in rapid thermal processing," IEEE Trans. Semiconduct. Manufact., vol. 7, pp. 34-41, Feb. 1994.

[18] G. L. Young and K. A. Mcdonald, "Effect of radiation shield angle on temperature and stress profiles during rapid thermal annealing," IEEE Trans. Semiconduct. Manufact., vol. 3, pp. 176-182, Nov. 1990.

[19] F. Y. Sorrel, M. J. Fordham, M. C. Öztürk, and J. J. Wortman, "Temperature uniformity in RTP furnaces," IEEE Trans. Electron Devices, vol. 39, no. 1, p. 75, Jan. 1992.
[20] S. A. Campbell and K. L. Knutson, "Transient effects in rapid thermal processing," IEEE Trans. Semiconduct. Manufact., vol. 5, pp. 302-307, Nov. 1992

[21] R. H. Perkins, T. J. Riley, and R. S. Gyurcsik, "Thermal uniformity and stress minimization during rapid thermal processes," IEEE Trans. Semiconduct. Manufact., vol. 8, pp. 272-279, Aug. 1995.

[22] J. R. Howell, Radiation Configuration Factor. New York: McGrawHill, 1982.

[23] A. N. Kolmogorov and S. V. Fomin, Introductory Real Analysis. New York: Dover, 1970

[24] SystemBuild/WS V2.4 User's Guide, MDG002-111.

[25] C. Hill, S. Jones, and D. Boys, "Rapid thermal annealing-Theory and practice," in Reduced Thermal Processing for ULSI, NATO ASI Series B: Physics, pp. 143-180, 1989.

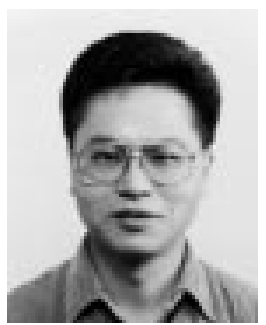

Yaw-Kuen Jan received the B.S. degree in electrical engineering from the National Taiwan University, Taipei, Taiwan, R.O.C., in 1986, and the M.S degree in control engineering from the National Chiao-Tung University (NCTU), Hsinchu, Taiwan, in 1990. He is currently pursuing the Ph.D. degree in control engineering at NCTU.

Since 1990, he has been a Vice-Engineer in the Chung-Shan Institute of Science and Technology. His research interests are in lamp configuration design and temperature control in rapid thermal processing.

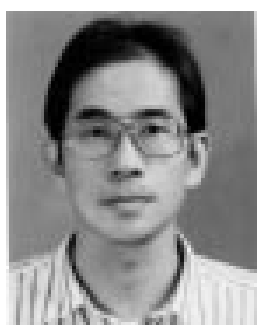

Ching-An Lin received the B.S. degree from the National Chiao-Tung University (NCTU), Hsinchu, Taiwan, R.O.C., in 1977, the M.S. degree from the University of New Mexico, Albuquerque, in 1980, and the Ph.D. degree from the University of California, Berkeley, in 1984, all in electrical engineering.

He was with the Chung-Shan Institute of Science and Technology from 1977 to 1979 and with Integrated Systems, Inc. from 1984 to 1986. Since June 1986, he has been with the Department of Control Engineering, NCTU, where he is a professor. His current research interests include multivariable control and signal processing. 